\title{
Análisis de los gastos operativos-financieros y su incidencia en la rentabilidad de empresa transporte
}

\author{
Analysis of operating-financial expenses and their impact on the profitability of the \\ transport company
}
Análise das despesas operacionais-financeiras e seu impacto na rentabilidade da transportadora

Recibido: octubre 2019

Arbitrado: noviembre 2019

Publicado: enero 2020
ABSTRACT

The objective of the study is to

El objetivo del estudio fue analizar los gastos operativos-financieros y su incidencia en la rentabilidad de la empresa Transporte Respalfianza, C.A. en el período 2015-2017. Los objetivos específicos consistieron en describir la estructura de costos operativos y financieros durante el período de estudio, analizar los Estados Financieros de la Empresa durante el período en cuestión utilizando las razones de rentabilidad financiera. Por otro lado, a nivel metodológico, la investigación fue cuantitativa y el diseño de investigación fue no experimental, apoyada en un diseño de campo. La metodología empleada estuvo constituida por un tipo de investigación documental y un nivel de carácter descriptivo. Se concluye que la empresa Transporte Respalfianza, C.A. en el período 2015-2017, tiene una estructura de costos muy poco definida, donde, los costos fijos representaron un promedio del 42,93\% y los costos variables promediaron el $39,11 \%$, con respecto a los ingresos.

Palabras Clave: Gastos operativos; financieros; costos; rentabilidad, costos analyze operating-financial expenses and their impact on the profitability of the company Transporte Respalfianza, C.A. in the period 2015-2017. The specific objectives consisted of describing the structure of operating and financial costs during the study period, analyzing the financial statements of the company during the period in question using the financial profitability ratios. On the other hand, at the methodological level, the research was quantitative and the research design was non-experimental, supported by a field design. The methodology used consisted of a type of documentary research and a descriptive level. It is concluded that the company Transporte Respalfianza, C.A. In the 2015-2017 period, it has a very poorly defined cost structure, where fixed costs represented an average of $42.93 \%$ and variable costs averaged $39.11 \%$, with respect to income.

Key words: Operating expenses; financial; costs; profitability, costs

\author{
《 Laura Hernández \\ lasangredecristo@gmail.com \\ ORCID: 0000-0002-6006-3770
}

Universidad de Carabobo, Venezuela

\section{RESUMO}

O objetivo do estudo é analisar as despesas operacionais-financeiras e seu impacto na rentabilidade da empresa Transporte Respalfianza, C.A. no período 2015-2017. Os objetivos específicos consistiram em descrever a estrutura dos custos operacionais e financeiros durante o período do estudo, analisando as demonstrações financeiras da empresa no período em questão através dos rácios de rentabilidade financeira. Por outro lado, a nível metodológico, a investigação foi quantitativa e o desenho de investigação não experimental, apoiado num desenho de campo. A metodologia utilizada consistiu em um tipo de pesquisa documental e um nível descritivo. Concluise que a empresa Transporte Respalfianza, C.A. No período 2015-2017 apresenta uma estrutura de custos muito mal definida, onde os custos fixos representaram em média $42,93 \%$ e os custos variáveis em média $39,11 \%$, no que respeita ao rendimento.

Palavras chave: Despesas operacionais; financeiro; custos; lucratividade, custos 


\section{INTRODUCCIÓN}

$\mathrm{E}$ mundo actual está caracterizado por factores como la globalización, los avances tecnológicos, la integración de los bloques económicos, así como múltiples cambios de orden, económico, social, cultural, político y hasta jurídico que rigen en la economía mundial y nacional de muchos países. Al respecto, Camacho (2015), señala que:

En el mundo se han desarrollado procesos de cambios importantes en lo político, social, económico y en lo cultural, aunque en su mayoría han propiciado el ahondamiento entre ricos y pobres con una brecha cada vez más marcada, originada por sus propios gobernantes que no han sabido dirigir la capacidad creadora de sus habitantes hacia el logro de un mejor nivel de vida en el plano social (p.17).

En tal sentido, en el contexto empresarial, a los fines de mantenerse en el mercado mundial tan acelerado y de firmes cambios se ha vuelto una necesidad para los directivos el desarrollo y gestión de capacidades en la entidad, además la adaptación al uso de tecnología de punta, amplias posibilidades de financiamiento y el manejo de información oportuna y veraz, así como también la inclusión en sus modelos administrativos de planteamientos estratégicos concretos hacen que sea necesario el que los directivos no sólo reconozcan y gestionen las capacidades existentes en la empresa, sino que además desarrollen con rapidez otras nuevas.

En esta perspectiva, las empresas deben enfrentar la creciente competencia, por lo cual tienen que desarrollar sus propias estrategias para ser más competitivas, de ahí la importancia de reducir al máximo posible los costos de producción a través del sistema de costos que permita controlar de manera adecuada los mismos, así como también la incidencia de los desperdicios para que estos no afecten en gran medida la estructura de costos. Es decir, que la determinación de los costos y el control adecuado de los mismos, reviste gran importancia para todas las empresas del sector transporte para hacer frente a la competencia, maximizando recursos y minimizando costos. Al respecto Molina (2003) refiere:

Para que las empresas logren la verdadera competitividad deben desarrollar y utilizar algunas técnicas de gestión, tendentes a controlar y reducir costos, así como a mantener su posicionamiento en el mercado, a través del liderazgo en costos y la diferenciación de los productos. (p. 25)

Lo anterior es cónsono con lo expresado por Osorio (1998), al indicar que las técnicas de gestión son procedimientos que se utilizan para optimizar la utilización de los recursos de la organización, para aumentar la competitividad. Estas técnicas se sustentan en nuevas tecnologías aplicadas al diseño y fabricación de bienes y servicios, cuya misión fundamental es "...hacer más competitivo un producto al tener un bajo costo de fabricación, al ser entregado en el momento 
oportuno, al aumentar su confiabilidad en la operación o al dar la solución a un problema específico del cliente" (Ramírez, 1997, p. 62), lo cual permite aumentar la eficiencia de las empresas, a través de información oportuna y confiable para la toma de decisiones y fijación de estrategias.

Cabe destacar, lo significativo que resulta para las empresas, el entorno económico donde se desenvuelven, particularmente las empresas venezolanas, ante la fuerte crisis económica que aqueja al país, ha sido un gran reto sostenerse en el tiempo, lo cual implica un alto nivel de incertidumbre. Cada día el efecto inflacionario incide en los costos operativos de estas empresas, ocasionando bajos niveles de ventas. Así mismo, estos problemas indican que se ha vuelto de suma necesidad, medir y controlar los costos para conocer cómo se han establecidos sus precios y determinar su rentabilidad para un mayor monitoreo de sus indicadores.

De ahí lo significativo de la rentabilidad de los costos que se ocasionan, comprende la revisión y análisis de los mismos, al respecto Gayle, (2009), destaca que:

Los administradores deberán usar el análisis de costos y las técnicas de control tan sólo cuando los beneficios anticipados que se producirán al ayudar a lograr las metas de la administración excedan a los costos. Este es el principal criterio para elegir entre enfoques contables alternativos y comúnmente se conoce como enfoque de costobeneficio. (p.4).

La falta de un sistema de costo adecuado, puede provocar que los costos no sean los idóneos para la adquisición de insumos, no se analizan cotizaciones para proceder a comprar al menor costo y dentro de las mejores condiciones, por lo que los precios de venta de cualquier producto o servicio son más altos que los de la competencia, lo que afecta las ventas, rentabilidad y los objetivos de crecimiento de la empresa.

Según Torres (2002), la contabilidad de costos se relaciona con la información de costos para uso interno de la gerencia y ayuda de manera considerable a la gerencia en la formulación de objetivos y programas de operación en la comparación del desempeño real con el esperado y en la presentación de informes. De allí que la importancia de establecer los costos en las organizaciones de cualquier índole, permiten tomar decisiones a un corto o largo plazo, a fin de obtener resultados significativos que favorezcan a la organización.

De esta forma, es importante señalar que este aspecto representa cifras imprescindibles para la determinación de la rentabilidad, ya que constituye la erogación económica que se hace dentro la entidad, que luego de su determinación, se puede establecer el precio de venta al público del bien o servicio en cuestión. En este caso, el costo está formado por el precio del servicio y el precio de la mano de obra directa e indirecta utilizada y demás gastos necesarios. Por lo tanto, el correcto análisis de los costos de producción permite conocer qué, dónde, cómo, cuándo y en qué medida realizo el servicio y, lo más importante es que otorga a la gerencia una base cierta de cálculo de los precios de venta para así generar auténticos beneficios a la empresa. 
La escogencia de un sistema que informe el verdadero costo el cual influye en la rentabilidad de las empresas es de vital importancia, tal es el caso de la problemática en la empresa del sector transporte, la empresa Transporte Respalfianza, C.A., dedicada al servicio de traslado de mercancía por todo el territorio nacional con una trayectoria de más de 10 años en el mercado, la cual cuenta con una flota de vehículos de carga pesada. La escasez de repuestos, la falta de divisas para importar los mismos y los altos precios de aquellos que puedan encontrarse en existencias, en el mantenimiento de las unidades ya existentes y aún más en aspirar realizar inversiones en nuevas unidades.

En este orden de ideas, se realizó una entrevista con el Gerente General de la empresa Transporte Respalfianza, C.A., quien manifestó su preocupación por la empresa, en la cual expuso, la situación país y que han disminuido los niveles de rentabilidad Además, se evidenció una inconsistencia en el cálculo de los costos, lo que puede estar incidiendo en las ventajas comparativas y competitivas, ya que al afectar la base para la fijación de los precios, éstos pudieran estar sobre o sub valuados, según sea el caso, lo cual a su vez pudiese estar limitando las estrategias de mercadeo y por ende, se pudiesen estar perdiendo oportunidades de negocios.

Adicional, la desviación en los costos y la falta de un valor razonable afecta la rentabilidad del servicio prestado, modificando los indicadores de gestión fundamentales para el análisis financiero de la empresa. En tal sentido, el margen bruto no mide eficazmente el porcentaje de cada unidad monetaria de cobro por el servicio, que queda después de deducir todos los costos incurridos, y distorsiona las tendencias y variaciones de la utilidad bruta, necesarias para la panificación financiera.

Según esta información, la empresa se ha visto afectada en el incremento de los costos, pese haber alcanzado aumento en los ingresos, las ganancias han permanecido sin mostrar mejoras significativas. Actualmente, los registros de los costos son llevados a cabalidad bajo la supervisión directa del Gerente de Administración, pero aun así, no existe un detalle minucioso, preciso y conciso que le provea a la Gerencia General determinar la estructura real de costos; por cuanto lo que se persigue es un estudio de los costos que se orienten hacia la optimización de los mismos y por consiguiente, se pueda ofrecer un servicio a bajo costo y de excelente calidad, dado que, a través del estudio se pueden establecer parámetros que permitan resolver los problemas con determinación, análisis, control y reducción de costos de producción, en la búsqueda de la mejora continua en la Gestión Estratégica Financiera, con miras a ser más competitivos.

En este sentido, la investigación tiene como objetivo principal elaborar un análisis de los gastos operativos-financieros y su incidencia en la rentabilidad de la empresa Transporte Respalfianza, C.A. en el período 2015-2017, lo que conlleva a Identificar la estructura de los costos operativos y financieros durante el período 2015-2017, examinar los estados financieros aplicando las razones de rentabilidad financiera durante el periodo 2015-2017, determinar la relación entre los costos y las utilidades a través de las técnicas estadísticas, analizar el comportamiento y la 
relación de los costos y la utilidad percibida por la empresa a través del punto de equilibrio y apalancamiento operativo.

La empresa Transporte Respalfianza, C.A., al igual que toda empresa del sector transporte, requiere de una adecuada gestión financiera que implica el conocimiento y manejo de sus costos de servicios, con el propósito de reportar a sus inversionistas niveles de rentabilidad que le sean satisfactorios. Específicamente en lo que ha sido la trayectoria de los costos y su rentabilidad en los últimos años, que no ha sido del todo satisfactoria, razón por lo cual se considera que la investigación reviste de importancia, por cuanto permitirá establecer cuál ha sido la evolución de dichas variables, y la incidencia de la primera (costos) sobre la segunda (rentabilidad).

En base a tal premisa se justifica la realización de la investigación, ya que ofrecerá un análisis de los gastos operativos-financieros y su incidencia en la rentabilidad de la empresa Transporte Respalfianza, C.A. en el período 2015-2017. Por otro lado, a nivel empresarial se justifica, ya que va dirigido a la parte de la alta gerencia de la empresa objeto de estudio, que tiene un gran compromiso con los accionistas, los empleados y la sociedad en general mediante un buen manejo de los recursos, este estudio le aportará un análisis crítico-razonable para asegurar la permanencia y las mejores prácticas para lograr el éxito en la organización, para ampliar sus conocimientos sobre los costos de producción y distribución para proyectar su rentabilidad, también podrá servir a aquellas personas que están interesadas en desarrollar actividades futuras en este tipo de empresas.

\section{MÉTODO}

$\mathrm{E}$ I estudio se enmarcó dentro de una investigación cuantitativa, descriptiva, de campo. Los datos fueron obtenidos de forma directa del caso de estudio, la empresa Transporte Respalfianza, C.A. en el período 2015-2017, y se realizó un estudio sistemático con el propósito de describirlo, analizarlo, explicando causas y efectos, así como su incidencia en la rentabilidad.

La técnica para recolección de información utilizada en este caso específico, a fin de preservar la objetividad de la investigación, fue la revisión documental, la misma consistió en la lectura de material bibliográfico como son documentos de la empresa, estados financieros y otros reportes contables. Se observó la incidencia en la rentabilidad de la empresa Transporte Respalfianza, C.A. en el período 2015-2017, para este caso de estudio, la oportunidad de analizar los Estados Financieros de la Empresa durante el período en estudio aplicando las razones de rentabilidad financiera; establecer la relación entre los costos y las utilidades a través de las técnicas estadísticas y analizar el comportamiento y la relación de los costos y la utilidad percibida por la empresa a través del punto de equilibrio y apalancamiento operativo. 
Se realizó posteriormente un análisis de datos estadístico descriptivo, a través de la distribución de las puntuaciones o frecuencias donde se expresó información relativa a los resultados alcanzados, indicando tanto la frecuencia en número, como la proporción expresadas en términos porcentuales. De igual manera, se realizó un análisis descriptivo en los estados financieros; para establecer la relación entre los costos y las utilidades a través de las técnicas estadísticas y analizar el comportamiento y la relación de los costos y la utilidad percibida por la empresa a través del punto de equilibrio y apalancamiento operativo.

\section{RESULTADOS}

\section{Generalidades}

Los estados de resultados sirvieron para el análisis de los gastos operativos-financieros y su incidencia en la rentabilidad de la empresa Transporte Respalfianza, C.A. en el período 2015-2017, y fueron preparados de acuerdo con el método de costeo absorbente, no obstante, para efectos de estudios, los mismos fueron estructurados de acuerdo con el método del costeo directo o variable. Ello con el fin de analizar de una manera más adecuada, la estructura y comportamiento de éstos.

\section{Renglones principales}

- Ingresos por Fletes: son las entradas de dinero que obtiene la empresa producto de los servicios prestados de transporte, fletes,

- Costo de Venta: en esta cuenta se registran las compras y salidas de los repuestos, cauchos, entre otros.

- Gastos Administrativos: son las erogaciones en que incurre la empresa para poder prestar sus servicios a sus clientes, las erogaciones más comunes son realizadas en el Gasto de personal, servicios externos e intereses financieros.

- Gastos Operacionales: se refiere a los suministros de oficinas y gastos de seguro.

- Utilidad del Ejercicio: es el margen obtenido por la empresa después de deducir los costos operativos y financieros a los ingresos por servicios, comúnmente se le conoce como la ganancia obtenida por la empresa en su ejercicio económico; el cual permite medir el manejo y rentabilidad de este ente comercial. 


\section{Estructura de costos de servicios durante el Período 20152017}

La estructura de costos permite cuantificar de forma monetaria, las erogaciones necesarias para prestar un servicio a un cliente determinado. La estructura de costo de la empresa Transporte Respalfianza, C.A. en el período 2015-2017, es la conformación de los conceptos y montos incluidos en los estados de resultados del período 2015-2017, y los mismos fueron reestructurados en base al método del costeo directo y se clasificó de acuerdo al comportamiento de los costos; ello con el fin de facilitar su análisis.

Sin embargo, éstos son clasificados en costos fijos y variables, por cuanto, los fijos se mantienen constante durante todos los ejercicios económicos, aunque se paralicen las actividades de la empresa y los variables se incurren solo si la empresa está prestando sus servicios médicoquirúrgicos.

La estructura de costos de la empresa Transporte Respalfianza, C.A. en el período 2015-2017, refleja que, en el año 2015, el costo total fue de Bs. 55.931.954,27, que los costos variables se ubicaron en un $84,68 \%$ del total de costo. Cabe mencionar, que los rubros que sobresalieron son el costo de venta con un $84,26 \%$, el gasto de personal con un $0,41 \%$ y gastos de mantenimiento con un $0,30 \%$ del costo total. Por cuanto, los costos fijos forman parte del costo total, ellos constituyeron el 15,32\%, el cual significó una diferencia muy representativa con respecto al costo variable, mientras que en los costos fijos las partidas más representativas fueron la de prestaciones sociales con un $4,21 \%$, seguido de gastos de seguros con $8,98 \%$, gastos de mantenimiento con $1,21 \%$ y sueldos y salarios con $0,46 \%$.

Para el año 2016, los costos fijos totalizaron $119.195 .286,69$ representando el $90.70 \%$ del costo total, las partidas más representativas fueron el costo de ventas con un $90,34 \%$, que mantiene una tendencia al alza con respecto al año anterior, mientras que aportes y retenciones representaron el $0,06 \%$ y los gastos de mantenimiento representaron el $0,23 \% \%$ del costo total. Sin embargo, los costos variables, los costos fijos ubicándose en un 15,32 \% del costo total. Se puede expresar que el costo de venta representa el $90,34 \%$ y los sueldos y salarios obtuvieron porcentualmente $0,13 \%$. Entre los motivos que originaron en incremento en los costos fijos, destaca la mayor el costo de ventas.

En el año 2017, los costos variables representaron el $82,12 \%$ del costo total que fue de Bs. $132.778 .205,25$, en los variables el costo de venta representa el $81,15 \%$ del total de costo y el 62,38 $\%$ del costo variable, el gasto de personal y los servicios públicos obtuvo una porción del 17,88 \% del total del costo variable de este año. Mientras que el costo fijo fue del $17,88 \%$ del costo total, el gasto de mantenimiento del total de costo, lo cual presenta una tendencia ascendente para el primero y segundo, y una tendencia descendente para el tercero con respecto al año anterior estudiado. 


\section{Análisis de los estados financieros de la empresa durante el periodo 20152017}

A efectos de llevar a cabo el análisis de los Estados Financieros de la Empresa Transporte Respalfianza, C.A., se procedió a calcular las razones financieras durante el período de estudio. Los datos son presentados en cuadros y gráficos y posteriormente comentados en forma global.

\section{Razón de ingresos netos entre activos (Rva)}

Al analizar la razón de ingresos netos sobre activos, permitió medir la eficiencia de cómo la empresa Transporte Respalfianza, C.A., manejan los activos de la empresa. En 2015 esta razón obtuvo un valor de 1,79. Para el 2016 la razón estudiada fue de 1,93, lo que refleja un aumento con respecto al 2015, debido a una leve alza en los ingresos y un aumento en los activos; mientras que en el año 2017 el resultado obtenido fue de 1,75, el cual bajo con relación al año 2016.

El resultado obtenido en la aplicación de esta razón de ingresos entre activos se considera óptima, puesto que, los niveles de ingresos por servicios se mantuvieron, a través de los ejercicios económicos estudiados, lo que demuestra el buen manejo de sus activos por parte de laempresa Transporte Respalfianza, C.A.

\section{Tasa de rendimiento del capital (Trc)}

Esta tasa mide la rentabilidad de los activos, sin importar las formas como fueron financiados por sus acreedores o accionistas.

El análisis de los resultados en este renglón arroja que, en el año 2015, el rendimiento del capital fue de $0,29 \%$, considerándose como bajo, con respecto al año 2016 , que fue de $0,21 \%$, No obstante, en el año 2017 esta medida representó el 0,31\% de los activos de la empresa Transporte Respalfianza, C.A., lo que permite decir, que obtuvo alto rendimiento en comparación con el año 2017, reflejando un nivel de rentabilidad ascendente. Pero a nivel general, esta empresa mantiene un nivel de rentabilidad aceptable que promedio un $0,27 \%$ en los tres años estudiados.

\section{Tasa de rendimiento del capital contable de los accionistas (TRCCA)}

Según el análisis en este aspecto, para el año 2015, la tasa de rendimiento del capital contable delos accionistas de la empresa Transporte Respalfianza, C.A., correspondiente es del 42,38\%. En el año 2016, esta medida obtuvo como resultado un 24,88 \% de su capital contable, muy inferior al año 2015, que promedió el 33,63\% de rendimiento para los accionistas, debido a una disminución de las utilidades operativas. En el ejercicio financiero del 2017, la tasa devengada por el capital invertido por los accionistas comunes fue de 41,10 \%, muy inferior al año 2015.

Al observarse los resultados obtenidos en el periodo 2015 2017, esta tasa promedio un 36,12\%, se podría decir, que la empresa Transporte Respalfianza, C.A es una empresa con un de nivel aceptable de rentabilidad para sus accionistas, a pesar de ser una rama de servicio. 


\section{CONCLUSIONES}

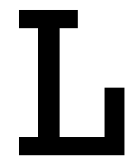

a empresa Transporte Respalfianza, C.A., S.A, cuenta con una estructura de costo bien definida, en donde se destacan como los rubros más importantes costo de venta, gasto de personal, gastos de mantenimiento y servicios externos. Al clasificar los costos en relación a su comportamiento, en comparación con los ingresos, se pudo encontrar que los costos variables representaron un promedio del $82,12 \%$, y los costos fijos promediaron el $17,88 \%$.

Al efectuar el análisis de los estados financieros mediante las razones de rentabilidad se puede puntualizar lo siguiente, la razón de ingresos sobre activos y la tasa de rendimiento del capital, mostró una tendencia ascendente en los años 2015, luego disminuyó en el 2016, y luego aumentó en el 2017, su inclinación fue ascendente debido aún aumento en los ingresos. Pero, a escala general estas medidas financieras promediaron un alto nivel de rentabilidad, lo cual, debería mantenerse y/o mejorarse en el futuro para un buen desenvolvimiento financiero de la empresa.

El resultado de la utilidad por acción común, permitió apreciar que el nivel de utilidad obtenida por los accionistas es aceptable, porque, aunque exista un aumento de utilidad, se mantiene un margen de utilidad por acción elevado. Al establecer la relación entre los costos, ingresos y utilidades a través de las diferentes técnicas estadísticas, se encontró los puntos siguientes:

El análisis del coeficiente de correlación entre los costos e ingresos fue positiva intenso, mientras que para los ingresos y utilidad la correlación fue positiva débil, lo que demuestra mayor intensidad entre la variable independiente ingresos y la variable dependiente costos. La aplicación de la técnica estadística coeficiente de determinación expresó la proporción que tiene los costos sobre los ingresos, en esta medida la proporción de los costos fue significativa con ingresos por servicios. No obstante, la utilidad fue significativo en proporción de los ingresos. Esta tendencia confirma a nivel estadístico los análisis sobre el elevado nivel de costo de la empresa en comparación a los ingresos.

Al analizar el comportamiento y relación entre los costos, utilidades e ingresos se pude resaltar que el punto de equilibrio de los ejercicios económicos estudiados presentó resultados bastante altos, los cuales superaron los ingresos de la empresa Transporte Respalfianza, C.A., lo que podría crear una situación de inconveniencia al momento de cubrir sus costos.

En relación a la estructura de costos se sugiere a la gerencia de la empresa Transporte Respalfianza, C.A., implementar un mecanismo que permita clasificar los costos que arroja el sistema de contabilidad financiera en variables y fijos; aparte que facilita el análisis de los mismos, puede ser utilizado para el control y en general para la toma de decisiones.

Evaluación de los Procesos Internos, deben medirse los procesos para determinar si cumplen con las expectativas, las necesidades y los requerimientos de cada departamento. Si efectivamente los sistemas y procesos presentan consistencia el requerimiento primordial es mantener el control sobre ellos. 
Se recomienda una presentación uniforme de formatos, para todas las áreas, que adapten a las necesidades de los Departamentos de Producción y Materiales, de una forma flexible.

Creación de planes de acción, las empresas del sector, deben contar para la planificación financiera presupuestaria con manual de normas y procedimiento por departamentos, que definan específicamente las tareas y funciones a realizar por cada departamento, que en forma programada permitirá agilizar la formulación la productividad de las empresas.

Con la finalidad de lograr el máximo aprovechamiento de los datos que aporta la contabilidad y que son presentados en los estados financieros, se aconseja a la dirección de esta empresa solicitar que sean calculadas y analizadas las razones financieras y prestar mayor atención a las razones de rentabilidad, a fin de velar por que se mejoren los rendimientos alcanzados.

Se recomienda que al realizar este tipo de estudio en cualquier empresa tomar en consideración el ajuste por inflación.

En vista que el análisis estadísticos entre los costos y los ingresos demostraron que existía una alta relación entre ellos, sé sugiere a la Gerencia y en particular a la Junta Directiva de esta empresa, efectuar un cuidadoso análisis de los conceptos y naturaleza de los costos, ya que de mantenerse esta tendencia, aun cuando lograra incrementar significativamente los ingresos, esta inclinación tendría poco efecto sobre las utilidades, puesto que, los costos se incrementarían en una misma proporción de los ingresos.

\section{REFERENCIAS}

Camacho, B. (2015). América Latina en la mira de las políticas públicas. Caracas. Trópicos

Gayle, R. (2009). Contabilidad y administración de costos. México. D.F. McGraw-Hill Interamericana Editores, $6^{\text {a }}$ edición

Molina, O. (2003). Nuevas Técnicas de Control y Gestión de Costos en Búsqueda de la Competitividad. Venezuela. Actualidad Contable FACES. Año 6 No.6, Enero-Junio 2003

Osorio, O. (1998). Tendencias Actuales de la Contabilidad de Gestión. Federación de Colegios de Contadores Públicos de Venezuela. Fondo Editorial del Contador Público Venezolano. Caracas. Venezuela.

Ramírez, D. (1997). Contabilidad Administrativa. 5a edición. Editorial Mc Graw - Hill. México

Torres, A. (2012). Contabilidad de costo, análisis para la toma de decisiones. Colección del taller: Serie Educación. Edición 2. Editorial McGraw-Hill 